\title{
Sozial-kognitive Fähigkeiten und Suizidalität: Eine Übersicht
}

\author{
Patrizia Thoma' und Tobias Teismann² \\ Neuropsychologisches Therapie Centrum, AG Klinische Neuropsychologie, Fakultät für Psychologie, Ruhr-Universität Bochum \\ Forschungs- und Behandlungszentrum für Psychische Gesundheit, Fakultät für Psychologie, Ruhr-Universität Bochum
}

\begin{abstract}
Zusammenfassung: Psychische Erkrankungen gehen mit einem erhöhten Suizidrisiko einher. Fehlende soziale Zugehörigkeit und Unterstützung, interpersonelle Konflikte und Einsamkeit gelten als zentrale Risikofaktoren für suizidales Erleben und Verhalten. In der vorliegenden Übersichtsarbeit wurden in den Datenbanken PubMed und ISI Web of Knowledge alle englisch- oder deutschsprachigen Originalarbeiten bis Juni 2020 gesichtet, die Emotionserkennung, Empathie und Theory of Mind in Zusammenhang mit Suizidgedanken und/oder Suizidversuchen untersuchen. Dabei wurden 15 relevante Veröffentlichungen identifiziert. Das Befundmuster stellt sich insgesamt als heterogen dar, legt jedoch nahe, dass sowohl beeinträchtigte Fähigkeiten in diesen Bereichen als auch eine verbesserte oder übertriebene Emotionserkennung/Theory of Mind mit Suizidalität in Zusammenhang stehen können.
\end{abstract}

Schlüsselwörter: Suizid, Interpersonale Theorie suizidalen Verhaltens, Soziale Kognitionen, Empathie, Theory of Mind, Emotionserkennung

Social cognition and suicidality: A review

Abstract: Psychiatric and neurological disorders are associated with increased suicide risk. A lack of social integration and support as well as interpersonal conflicts and loneliness are commonly regarded as prominent risk factors for both suicidal ideation and behavior. In this review. we reviewed all empirical studies published in English or German and listed in PubMed or ISI Web of Knowledge up through June 2020 and included those that investigated, on the one hand, the relationship between emotion recognition, empathy, and theory of mind and suicidal ideation or behavior, on the other hand. Fifteen relevant papers were identified. The overall pattern is rather heterogeneous but suggests that both impaired abilities and improved or excessive emotion attribution/theory of mind may be associated with suicidal tendencies.

Keywords: suicide, interpersonal theory of suicidal behavior, social cognition, empathy, theory of mind, emotion recognition

\section{Einleitung}

Im Jahr 2019 starben in der Bundesrepublik Deutschland 9041 Menschen durch einen Suizid (Statistisches Bundesamt, 2021). Suizide werden über die gesamte Lebensspanne hinweg vollzogen und betreffen sowohl Frauen als auch Männer. Weltweit ist in den vergangenen Jahrzehnten ein substanzieller Rückgang der Suizidrate zu konstatieren - gleichwohl gehören Suizide weiterhin zu den häufigsten Todesursachen (Naghavi \& Global Burden of Disease Self-Harm Collaborators, 2019). Suizidversuche im Laufe des Lebens werden von 1.7\% der Deutschen berichtet (Nock, Borges \& Ono, 2012) und $8 \%$ der Befragten gaben in einer deutschen Repräsentativuntersuchung an in den vergangenen zwei Wochen unter ernsthaften Suizidgedanken gelitten zu haben (Forkmann, Brähler, Gauggel \& Glaesmer, 2012). Fehlende soziale Zugehörigkeit und Unterstützung sowie interpersonelle Konflikte und
Einsamkeit gelten als zentrale Risikofaktoren für suizidales Erleben und Verhalten (Heilbron, Franklin, Guerry \& Printstein, 2014; McClelland, Evans, Nowland, Ferguson \& O'Connor, 2020). In diesem Sinne zeigte sich beispielsweise in zwei groß angelegten amerikanischen Längsschnittstudien, dass ein höherer Grad sozialer Integration mit einer geringeren Suizidrate assoziiert ist - und dies sowohl bei Männern (Tsai, Lucas, Sania, Kim \& Kawachi, 2014) als auch bei Frauen (Tsai, Lucas \& Kawachi, 2015). Psychologische Autopsiestudien an jugendlichen und erwachsenen Suizidopfern verweisen überdies darauf, dass geringe soziale Unterstützung, Trennungen und das Erleben von Einsamkeit mit Suiziden assoziiert sind (Appleby, Cooper, Amos \& Faragher, 1999; Beautrais, 2002; Waern, Rubenowitz \& Wilhelmson, 2003). Vor diesem Hintergrund erstaunt es nicht, dass die Bedeutung sozialen Zugehörigkeitserlebens in aktuellen Theorien zum Verständnis suizidalen Erlebens und Verhaltens be- 
sonders betont wird (Joiner, 2005; Klonsky \& May, 2015; O'Connor \& Kirtley, 2018). Im Rahmen der Interpersonalen Theorie suizidalen Verhaltens postuliert beispielsweise Joiner (2005), dass die Wahrnehmung, nicht Teil einer wertgeschätzten Gruppe zu sein (thwarted belongingness), und der Eindruck, eine Belastung für andere darzustellen (perceived burdensomeness), assoziiert sind mit dem Wunsch, zu sterben. Der Theorie zufolge sind perceived burdensomeness und thwarted belongingness proximale, kausale und interagierende Risikofaktoren, deren Vorhandensein eine zwingende Voraussetzung für Suizidgedanken darstellt. In Studien ließ sich insbesondere die Bedeutung des Eindrucks, eine Last für andere Menschen zu sein, sehr konsistent nachweisen (Chu et al., 2017).

Deutlich seltener untersucht wurde bislang jedoch, welche kognitiven Faktoren fehlendem sozialem Zugehörigkeitserleben bei suizidalen Personen zugrunde liegen. Es ist beispielsweise denkbar, dass Defizite in der Emotionserkennung zu einem niedrigen Grad an sozialer Verbundenheit und Zugehörigkeit führen oder dass eine Beeinträchtigung der Empathiefähigkeit die Berücksichtigung der verheerenden Auswirkungen des eigenen Suizids untergraben könnte.

\section{Soziale Kognitionen}

Die Fähigkeit, Emotionen zu erkennen und sich in andere Menschen mental und emotional hineinzuversetzen, wird unter dem Oberbegriff „,soziale Kognitionen“ geführt. Darunter versteht man eine Vielzahl von psychologischen Prozessen, die im Rahmen sozialer Interaktionen relevant sind und sowohl basale Konstrukte, wie die Emotionserkennung, als auch komplexere Fähigkeiten, wie Empathie, Theory of Mind (ToM) und soziales Problemlösen umfassen (Adolphs, 2001; Beer \& Ochsner, 2006).

Als zentrale Aspekte, die für das Erleben von sozialer Ausgrenzung im Kontext suizidaler Krisen bedeutsam erscheinen, sollen in der aktuellen Übersichtsarbeit Emotionserkennung, Empathie und ToM betrachtet werden. Beide zuletzt genannten Konstrukte zielen auf die Dekodierung von emotionalen bzw. mentalen Zuständen anderer Personen $a b$ und hängen auf einer basalen Ebene von der Fähigkeit ab, emotionale Zustände anhand von fazialen und prosodischen Hinweisreizen sowie der Körperhaltung zu erkennen. Typische Aufgabenstellungen aus dem experimentellen und klinischen Untersuchungskontext erfordern das Benennen eines emotionalen (Gesichts-) Ausdrucks oder die Diskrimination zwischen verschiedenen Gesichtsausdrücken bzw. den dargestellten Intensitätsgraden einer Emotion, wie z.B. in Subtests der Penn PC-gestützten Neurokognitiven Testbatterie (Gur et al., 2001). An dieser Fähigkeit zur Emotionserkennung sind weit verzweigte neuronale Netzwerke beteiligt, die u.a. die Amygdala sowie orbitofrontale, frontoparietale und occipitotemporale Kortexanteile einbeziehen (Adolphs, 2001), wobei einzelne Emotionen mit spezifischen neuronalen Korrelaten assoziiert zu sein scheinen (Vytal \& Hamman, 2010). Auf einer übergeordneten Ebene ermöglicht Empathie, dekodierte Gefühlszustände anderer Personen aus deren Perspektive zu verstehen (kognitive Empathie) sowie emotional nachzuvollziehen und mit eigenen affektiven Reaktionen zu beantworten (affektive Empathie; Preston \& de Waal, 2002; Shamay-Tsoory, Aharon-Peretz \& Perry, 2009). Eine Untersuchung der affektiven Empathie erfolgt bislang meist - recht unbefriedigend - über Selbstberichtsmaße, wie z.B. den Interpersonal Reactivity Index von Davis (1980), und könnte valider am ehesten indirekt über psychophysiologische Parameter wie die Erfassung der Hautleitfähigkeit erschlossen werden, was jedoch in bisherigen Untersuchungen nur selten erfolgt. Die kognitive Komponente der Empathie überlappt mit affektiven Aspekten der ToM, welche ebenfalls das Erschließen emotionaler Zustände anderer im Sinne einer kognitiven Perspektivübernahme ohne eigene Gefühlsbeteiligung ermöglicht. Ein Testverfahren, welches klassischerweise zur Erfassung der kognitiven Empathie/ affektiven ToM verwendet wird, ist der Reading the Mind in the Eyes Test (Baron-Cohen, Wheelwright, Hill, Raste \& Plumb, 2001), in welchem komplexe Emotionen (z. B. Eifersucht) anhand der Augenpartie erkannt werden müssen. Kognitive Aspekte der ToM beziehen sich hingegen auf die Fähigkeit, die Gedanken, Überzeugungen und Intentionen von Interaktionspartner_innen zu verstehen und zu antizipieren (Shamay-Tsoory \& Aharon-Peretz, 2007). Untersucht wird diese Fähigkeit häufig, indem den Proband_innen Geschichten vorgelegt werden (z.B. Strange Stories Test: Happé, 1994), im Rahmen derer sie die Gedanken und Intentionen der Hauptperson, die sich bei höherem Schwierigkeitsgrad auch auf die Gedanken dritter Personen beziehen können, erschließen müssen.

ToM wird in der Literatur häufig synonym mit dem Begriff „mentalisieren“ verwendet. Entsprechend werden in der aktuellen Übersichtsarbeit die Begriffe kognitive Empathie/affektive ToM sowie kognitive ToM/mentalisieren gleichbedeutend verwendet werden. Den an dieser Stelle deutlich gewordenen Abgrenzungsschwierigkeiten der Konstrukte entsprechend, werden für Empathie und ToM zum Teil überlappende neuronale Korrelate identifiziert. Grob zusammengefasst scheint es, als würden die affektiven Aspekte von Empathie und ToM eher die ventralen Anteile frontotemporaler Kortizes und die kognitive Aspekte eher die dorsalen Areale involvieren (Gallese, 2003; Shamay-Tsoory, 2011).

Zusammenfassend ermöglichen soziale Kognitionen, zu verstehen, was in anderen Personen vorgeht, ihr Ver- 
halten im Situationskontext zu begreifen und auf dieser Basis eigenes Verhalten in sozialen Situationen zielgerichtet anzupassen (Henry et al., 2016). Diese Darstellung verdeutlicht bereits, dass auch weitere, nichtsoziale kognitive Prozesse, wie Gedächtnis und exekutive Funktionen, einen wichtigen Beitrag zu sozialen Kognitionen leisten (Channon, 2004; Kennedy \& Adolphs, 2012; ShamayTsoory, 2011). Insbesondere die Fähigkeiten, multiple (soziale) Informationen online $\mathrm{zu}$ verarbeiten (Arbeitsgedächtnis), flexibel zwischen der eigenen und einer fremden mentalen und emotionalen Perspektive zu wechseln (kognitive Flexibilität) und die eigene emotionale Perspektive zu unterdrücken (Inhibition), wurden in verschiedenen Studien mit soziokognitiven Fähigkeiten wie Empathie und ToM assoziiert (z. B. Eslinger, Moore, Anderson \& Grossman, 2011; Ze, Thoma \& Suchan, 2014). Ebenso bildet die Fähigkeit, sich mental in Vergangenheit und Zukunft zu projizieren, also im Wesentlichen mentales Zeitreisen, das auf episodischen Gedächtnisfunktionen beruht, eine Basis für die Fähigkeit, sich in andere mental und emotional hineinzuversetzen (Gaesser, 2020). Dementsprechend findet sich auch teilweise eine Überlappung der neuronalen Netzwerke, die (sozio)kognitive Funktionen vermitteln. So haben sich der Temporallappen, welcher für die Wahrnehmung sozialer Signale zuständig ist, sowie die Amygdala und somatosensorische, orbitofrontale und cinguläre Kortexanteile, welche die Verbindung dieser Wahrnehmungseindrücke mit weiteren motivationalen und kognitiven Prozessen herstellen, als besonders relevante gemeinsam genutzte Netzwerkknoten herauskristallisiert (Adolphs, 2001). Für die ausführlichere Darstellung von Modellen soziokognitiver Verarbeitung in Interaktion mit Prozessen der Wahrnehmung, der Aufmerksamkeit, des Gedächtnisses und ihrer Modulation durch interindividuelle Unterschiede, bezogen z. B. auf Alter, Geschlecht und Persönlichkeitseigenschaften, sei auf die integrative Darstellung von Dolcos et al. (2020) verwiesen.

\section{Soziale Kognitionen und Suizidalität}

Die Bedeutung von Einschränkungen sozialer Kognitionen für die Entwicklung, Aufrechterhaltung und Exazerbation von psychischen Erkrankungen wird seit Langem diskutiert - und dies auch mit Blick auf suizidales Erleben und Verhalten (z.B. Brüne, 2005; Kornreich et al, 2002). Die Suizidrate ist sowohl bei affektiven Störungen als auch im Rahmen von psychotischen Störungen deutlich erhöht (vgl. Gournellis et al., 2018). Für diese Erkrankungen existieren zahlreiche Befunde, welche Defizite im Bereich sozialer Kognitionen belegen (für eine Übersicht siehe Thoma, Friedmann \& Suchan, 2013). Grob ergibt sich folgendes Bild: Während in Zusammenhang mit depressiven Erkrankungen und auch bei der bipolaren Störung Einschränkungen der basalen Emotionserkennung (z. B. Cusi, Nazarov, Holshausen, Macqueen \& McKinnon, 2012) sowie eine erhöhte persönliche Betroffenheit auf affektiv-empathischer Ebene recht konsistent belegt werden konnten, ist die Befundlage für kognitive Empathie und ToM weniger eindeutig (vgl. Thoma et al., 2013). Psychotische Erkrankungen wie die Schizophrenie gehen eher mit globalen Beeinträchtigungen sowohl der Emotionserkennung, der Empathie als auch der ToM einher (z.B. Derntl et al., 2009; Vucurovic, Caillies \& Kaladjian, 2020); die Defizite erscheinen zudem stärker ausgeprägt $\mathrm{zu}$ sein als die von Patient_innen mit bipolaren und depressiven Erkrankungen (Derntl, Seidel, Schneider \& Habel, 2012). Auffällig ist auch, dass neurofunktionelle Veränderungen bei Menschen mit suizidalem Erleben und Verhalten in zentralen Anteilen derjenigen frontolimbischen Schaltkreise gefunden wurden, die, wie oben ausgeführt, mit soziokognitiven Funktionen in Verbindung gebracht wurden, u.a. den orbitofrontalen und anterior cingulären Kortizes (Auerbach, Pagliaccio, Allison, Alqueza \& Alonso, 2021).

Trotz des nicht immer klaren Musters einzelner Komponenten soziokognitiver Beeinträchtigungen bei den genannten psychischen Störungen wird deutlich, dass Defizite sozialer Kognitionen bei diesen Erkrankungen vorliegen und damit möglicherweise auch suizidales Erleben und Verhalten beeinflussen. Bislang wurde dieser Zusammenhang jedoch nur in relativ wenigen Studien untersucht. Einzig in Bezug auf soziales Problemlösen liegt etwa ein Dutzend Studien vor, welche überwiegend zu dem Schluss kommen, dass das Suizidrisiko bei verschiedenen Störungen in Zusammenhang mit Einschränkungen sozialer Problemlösefertigkeiten im Sinne maladaptiver Problemlösestile erhöht ist (vgl. D'Zurilla, Chang, Nottingham \& Faccini, 1998; Speckens \& Hawton; 2005). Dabei wurden soziale Problemlösefertigkeiten häufig im Selbstbericht und nicht mit Maßen zur Untersuchung von Verhalten und Fähigkeiten untersucht (z. B. auch Breitborde, Wastler, Pine \& Moe, 2020; Kwok, 2014).

Vor dem Hintergrund der enormen Bedeutung sozialer Faktoren für das Verständnis suizidalen Erlebens und Verhaltens besteht das Ziel des vorliegenden Artikels darin, die Literatur zum Zusammenhang zwischen Suizidalität und sozialen Kognitionen bzw. den soziokognitiven Konstrukten Emotionserkennung, Empathie und ToM zusammenfassend darzustellen. Wir vermuteten basierend auf den oben dargestellten Ausführungen systematische $\mathrm{Zu}-$ sammenhänge zwischen veränderten soziokognitiven Leistungen und erhöhter Suizidalität - und dies unabhängig vom ätiologischen Kontext. 


\section{Methodik}

Unter Verwendung der Datenbanken PubMed und Web of Knowledge wurde eine Literaturrecherche mit folgenden Suchstrings durchgeführt: (suicid* OR self harm OR self injur*) AND (theory of mind OR social cognition OR [emot* AND recognition] OR [affect* AND recognition] OR mentali* OR empath*). Des Weiteren wurden die Literaturverzeichnisse einschlägiger Publikationen nach relevanten Studien durchgesehen. Die Recherche schließt alle Untersuchungen ein, die bis zum 05.06.2020 publiziert wurden und in deutscher oder englischer Sprache veröffentlicht wurden. Grundsätzlich wurden nur Studien berücksichtigt, die folgenden Kriterien entsprachen: (1.) Es handelte sich um empirische Studien, keine Übersichtsarbeiten. (2.) Es wurde ein Verhaltens-/Fähigkeitsmaß zur Erfassung von Fähigkeiten im Bereich der Emotionserkennung, empathischem Einfühlungsvermögen oder ToM eingesetzt. Studien, in denen nur Selbstberichtsmaße (Fragebögen) verwendet wurden, wurden nicht berücksichtigt. (3.) Es wurde ein Zusammenhang hergestellt zu einem Maß für suizidales Erleben und Verhalten (Suizidgedanken, Suizidpläne, Suizidversuche, Suizide). Studien, im Rahmen derer ausschließlich nichtsuizidales selbstverletzendes Verhalten untersucht wurde, wurden nicht berücksichtigt. (4.) Die Studie wurde auf Englisch oder auf
Deutsch in einer Fachzeitschrift mit Peer-Review-Verfahren publiziert. Beide Autor_innen dieses Artikels gingen alle Treffer im Hinblick auf die definierten Suchkriterien zunächst unabhängig voneinander durch; eventuelle Diskrepanzen wurden anschließend bis zur Übereinstimmung geklärt. Die Ergebnisse der Literaturrecherche wurden in narrativer Weise aufbereitet und interpretiert.

\section{Ergebnisse}

Tabelle 1 bietet einen Überblick über die Studien, welche die Einschlusskriterien erfüllten. Insgesamt wurden zunächst in der Literaturdatenbank PubMed 914 Artikel aus dem Zeitraum von 1960 bis zum 05.06.2020 gemäß den oben dargestellten Suchkriterien identifiziert. Die mit identischen Suchkriterien durchgeführte Suche in der Datenbank Web of Knowledge ergab, bereinigt um Duplikate, 493 zusätzliche Treffer. Unter Anwendung der zuvor genannten Einschlusskriterien wurden 15 Studien in die Übersichtsanalyse eingeschlossen, alle in englischer Sprache.

In der folgenden Darstellung wird die Studienlage getrennt für die verschiedenen Teilkonstrukte sozialer Kognitionen dargestellt.

Tabelle 1. Übersicht über die Charakteristika der in die Literaturanalyse eingeschlossenen Studien $(N=15)$

\begin{tabular}{|c|c|c|c|c|}
\hline $\begin{array}{l}\text { Autor_innen/ } \\
\text { Jahr }\end{array}$ & Stichprobe(n) & $\begin{array}{l}\text { Operationalisierung Emoti- } \\
\text { onserkennung und ToM/Em- } \\
\text { pathie }\end{array}$ & $\begin{array}{l}\text { Operationalisierung } \\
\text { Suizidalität }\end{array}$ & Zentrale Ergebnisse \\
\hline $\begin{array}{l}\text { Barzilay et } \\
\text { al., } 2019\end{array}$ & $\begin{array}{l}\text { Daten von } 6151 \text { Kindern und } \\
\text { Jugendlichen (11 bis } 21 \text { Jahre) } \\
\text { aus der „Penn Neurodevelop- } \\
\text { mental Cohort“, zuvor gentypi- } \\
\text { siert, } 54.9 \% \text { weiblich, keine } \\
\text { besonderen (Vor-)Erkrankun- } \\
\text { gen } \\
\text { darunter } 672 \text { mit Suizidgedan- } \\
\text { ken }\end{array}$ & $\begin{array}{l}\text { Emotionserkennung: } \\
\text { zwei Teilaufgaben aus der } \\
\text { Penn Computerized Neuroco- } \\
\text { gnitive Battery: Differenzie- } \\
\text { rung zwischen Emotionskate- } \\
\text { gorien und } \\
\text { Emotionsintensitäten }\end{array}$ & $\begin{array}{l}\text { Bejahung eines einzel- } \\
\text { nen suizidassoziierten } \\
\text { ltems („Hast Du zu ir- } \\
\text { gendeiner Zeit viel dar- } \\
\text { über nachgedacht, Dich } \\
\text { umzubringen?“) }\end{array}$ & $\begin{array}{l}\text { keine signifikanten Unter- } \\
\text { schiede bezüglich der Leis- } \\
\text { tungen in den beiden Emoti- } \\
\text { onserkennungsaufgaben } \\
\text { zwischen Jugendlichen mit } \\
\text { Suizidgedanken vs. ohne, } \\
\text { wenn diese nach Geschlecht } \\
\text { und zusätzlich Pubertätssta- } \\
\text { tus (prä/post) aufgeteilt wur- } \\
\text { den }\end{array}$ \\
\hline $\begin{array}{l}\text { Brick et al., } \\
2019\end{array}$ & $\begin{array}{l}\text { Gentypisierungsdaten von } \\
9267 \text { US-amerikanischen Ju- } \\
\text { gendlichen ( } 8 \text { bis } 21 \text { Jahre) aus } \\
\text { der „Penn Neurodevelopmen- } \\
\text { tal Cohort“, aufgrund körperli- } \\
\text { cher Erkrankungen stationär } \\
\text { behandelt } \\
\text { 17\% mit Suizidgedanken aktu- } \\
\text { ell oder in Vorgeschichte }\end{array}$ & $\begin{array}{l}\text { Emotionserkennung: } \\
\text { zwei Teilaufgaben aus der } \\
\text { Penn Computerized Neuroco- } \\
\text { gnitive Battery: Differenzie- } \\
\text { rung zwischen Emotionskate- } \\
\text { gorien und } \\
\text { Emotionsintensitäten }\end{array}$ & $\begin{array}{l}\text { Bejahung von mind. ei- } \\
\text { nem von drei suizidas- } \\
\text { soziierten Items aus der } \\
\text { Kiddie-Schedule for Af- } \\
\text { fective Disorders and } \\
\text { Schizophrenia (Lebens- } \\
\text { zeit: viele Gedanken an } \\
\text { Tod oder Sterben und/ } \\
\text { der Nachdenken über } \\
\text { Suizid. In den letzten } 5 \\
\text { Monaten: Gedanken ans } \\
\text { Sterben, Tod oder Sui- } \\
\text { zid) }\end{array}$ & $\begin{array}{l}\text { schnellere und genauere } \\
\text { Emotionsidentifikation bei } \\
\text { Jugendlichen mit Suizidge- } \\
\text { danken im Vergleich zu den- } \\
\text { jenigen ohne }\end{array}$ \\
\hline
\end{tabular}




\begin{tabular}{llll}
\hline $\begin{array}{l}\text { Autor_innen/ Stichprobe(n) } \\
\text { Jahr }\end{array}$ & $\begin{array}{l}\text { Operationalisierung Emoti- } \\
\text { onserkennung und ToM/Em- } \\
\text { pathie }\end{array}$ & $\begin{array}{l}\text { Operationalisierung } \\
\text { Suizidalität }\end{array}$ & Zentrale Ergebnisse \\
\hline
\end{tabular}

Canal-Rivero 65 in Spanien stationär behan- ToM :

et al., 2017 delte Pat. (14-54 Jahre alt, $M=26,17$ Jahre) mit der ersten Episode einer Psychose aus dem schizophrenen und affektiven Formenkreis

Baseline zum Behandlungszeitpunkt + katamnestische Erhebung von Suizidalität nach 6 und 12 Monaten

bei $30.8 \%$ Suizidversuch innerhalb von 12 Monaten zwei „false belief“-Geschichten erster (falsche Überzeugung beim Protagonisten) und zweiter (falsche Überzeugung des Protagonisten über den mentalen Zustand einer anderen Person) Ordnung

„Hinting"-Task: In 10 Geschichten die wahren Absichten aus indirekter Rede erschließen
Frage nach Suizidversuchen im „Schedules for Clinical Assessment of Neuropsychiatry"; bei Bejahung Suizidversuch definiert als Selbstverletzung mit oder ohne ernsthafte Folgen mehr ToM-Fehler erster und zweiter Ordnung bei Pat. mit Suizidversuchen im Vergleich zu Pat. ohne Suizidversuche innerhalb von 12 Monaten

Vorhersage von Suizidversuchen innerhalb des Nachuntersuchungszeitraums allein durch ToM-Fehler erster Ordnung als von anderen Einflüssen unabhängigem Prädiktor $\begin{array}{ll}\text { Canal-Rivero } & \text { Zweitveröffentlichung zu Ca- } \\ \text { et al., 2019 } & \text { nal-Rivero et al., 2017 (s.o.) }\end{array}$

\section{ToM :}

zwei „false belief“-Geschichten erster (falsche Überzeugung beim Protagnisten) und zweiter (falsche Überzeugung des Protagonisten über den mentalen Zustand einer anderen Person) Ordnung
Frage nach Suizidversuchen im Interviewverfahren „Schedules for Neuropsychiatry"; bei Bejahung Suizidversuch definiert als Selbstverletzung mit oder ohne ernsthafte Folgen (Wert von 2 oder 3) Clinical Assessment of

mehr Suizidversuche innerhalb von 12 Monaten bei Pat., die ToM-Fehler erster, als auch bei Pat. die ToMFehler zweiter Ordnung machten im Vergleich zu fehlerfrei Antwortenden

nur ToM-Fehler erster Ordnung prädiktiv für die Anzahl der Suizidversuche innerhalb von 12 Monaten

Pat. mit aktuellen Suizidgedanken genauer beim Erkennen negativer Emotionen bei dynamischen Stimuli als Pat. ohne Suizidgedanken

Einschätzungen des negativen Affektes für dynamische Stimuli an T1 nicht prädiktiv für Suizidgedanken an T2

dynamische Stimuli:Bell Lysaker Emotion Recognition Task:Videoclips, in denen der männliche Protagonist mittels Prosodie, Gesichts- und Oberkörperausdruck eine der sechs Basisemotionen oder einen neutralen Ausdruck zeigt keine signifikanten Befunde für statische Stimuli
Duño et al., 2009

\author{
57 Pat. mit Schizophrenie \\ ( $M=31,5$ Jahre $\pm 7,8$ Jahre), in \\ Spanien ambulant behandelt, \\ in Remission \\ Einteilung der Pat. in solche

\section{ToM} \\ zwei false-belief Geschichten \\ zu falschen Überzeugungen \\ ersten Grades und zwei zu \\ falschen Überzeugungen \\ zweiten Grades
} mit mind. einem Suizidversuch (43.9\%) in der Vorgeschichte und solche ohne Suizidversuch medizinische Unterlagen und Interviews mit Angehörigen zur Erfassung früherer Suizidversuche
Beeinträchtigungen der ToM zweiter Ordnung bei Pat. mit lebenszeitlichen Suizidversuchen im Vergleich zu Pat. ohne Suizidversuch, allerdings bei gleichzeitig beeinträchtigtem IQ

Vorhersage von Suizidversuchen in Vorgeschichte spezifisch durch ToM-Fehler zweiter Ordnung, auch nach Kontrolle für $I Q$ und andere Variablen 


\begin{tabular}{llll}
\hline $\begin{array}{l}\text { Autor_innen/ Stichprobe(n) } \\
\text { Jahr }\end{array}$ & $\begin{array}{l}\text { Operationalisierung Emoti- } \\
\text { onserkennung und ToM/Em- } \\
\text { pathie }\end{array}$ & $\begin{array}{l}\text { Operationalisierung } \\
\text { Suizidalität }\end{array}$ & Zentrale Ergebnisse \\
\hline
\end{tabular}

$\begin{array}{lll}\text { Harenski et } & 126 \text { männliche Personen, } & \text { Empathie: } \\ \text { al., 2017 } & \text { U.S.A., (18-60 Jahre), darunter } & \text { Videos, in denen Protagonis- } \\ & \text { a) } 41 \text { forensische Pat. mit Er- } & \text { ten von realen emotionalen } \\ \text { krankungen mit psychotischen } & \text { Erlebnissen erzählen. Pat. } \\ & \text { Merkmalen (Schizophrenie, } & \text { wählen aus einer Liste Emoti- } \\ \text { schizo-affektive Störung, bipo- } & \text { onen aus, von denen sie glau- } \\ \text { lare Störung, depressive Stö- } & \text { ben, dass die Hauptperson sie } \\ \text { rung), davon 18 nach Suizidver- } & \text { erlebt hat und ordnen diese } \\ \text { such b) 59 Straffällige ohne } & \text { nach Relevanz. „Empathische } \\ \text { psychotische Erkrankung c) 26 } & \text { Genauigkeit“ als Grad der } \\ \text { strafrechtlich und psychiat- } & \text { Übereinstimmung mit den } \\ \text { risch unauffällige Kontrollen } & \text { tatsächlich von den Protago- } \\ \text { (M Alter in den 4 Gruppen: } & \text { nisten angegebenen Emotio- } \\ \text { 32.5-40.2 Jahre) } & \text { nen }\end{array}$

Hatkevich et in den U.S.A. stationär psychi-

al., 2019 atrisch behandelte jugendliche Pat. (12-17 Jahre, M: 15.4 Jahre), 14 verschiedene Diagnosen, davon $50.1 \%$ eine affektive Störung, $52.2 \%$ eine Angststörung, $39.4 \%$ eine externalisierende Störung

$34 \%$ Suizidgedanken in den letzten 4 Wochen und $50.7 \%$ im letzten Jahr

$32.9 \%$ Suizidversuch im letzten Jahr, $42.2 \%$ mind. einen Versuch im Leben

\begin{tabular}{ll}
\hline Ortuño-Sier- & 1509 mexikanische Schüler_- \\
ra et al., & innen (14-19 Jahre, M: 16,5) \\
2020 & aus einem zufällig ausgewäh- \\
& ten, stratifizierten Cluster von \\
& 15.000 \\
& \\
& erhöhtes Suizidrisiko bei 83 \\
& Schüler_innen
\end{tabular}

Paradiso et retrospektive Analyse der Da-
al., 2016 ten von 185 ( $M=58$,7 Jahre $\pm 3.49)$ US-Vietnam-Veteranen mit verschiedenen psychiatrischen Diagnosen (davon 131 nach Kopfverletzungen), die zum Untersuchungszeitpunkt Suizidgedanken angaben $(\mathrm{N}=46)$ oder nicht $(\mathrm{N}=139)$

Richard-De- 35 aktuell nicht-depressive vantoy et al., Pat. nach einem schwerwie2013 genden Suizidversuch und affektiven Störungen in Vorgeschichte, 31 aktuell nicht-depressive Pat. mit affektiven Störungen aber ohne Suizidversuch in der Vorgeschichte, 37 gesunde Kontrollpersonen, alle 21-62 Jahre alt $(\approx M=40-44)$

\section{ToM :}

Movie for the Assessment of

Social Cognition: ToM-Fragen zu Filmsituationen, vier Indizes: keine ToM, weniger ToM, akkurate ToM, ,exzessive“ToM

medizinische Unterlagen zur Erfassung früherer Suizidversuche

Columbia Suicide Severity Rating Scale

PC-gestützte Erhebung mithilfe des Computerized Diagnostic Interview Schedule for Children von Suizidgedanken in den letzten 4 Wochen und im letzten Jahr und von Suizidversuchen im letzten Jahr und lebenszeitlich verminderte empathische Genauigkeit bei psychotischen Forensikpat. mit Suizidversuchen in der Vorgeschichte im Vergleich zu denen ohne Suizidversuche, den nicht-psychotischen Straffälligen und den Kontrollpersonen

Zusammenhang zwischen verminderter empathischer Genauigkeit und einem erhöhten Risiko für vergangene Suizidversuche

Tendenz zur übertriebenen Zuschreibung mentaler Zustände (,exzessive ToM“) hängt signifikant mit Suizidgedanken in den letzten 4 Wochen und Suizidversuchen im letzten Jahr zusammen
Emotionserkennung:
zwei Teilaufgaben aus der
Penn Computerized Neuroco-
gnitive Battery: Differenzie-
rung zwischen Emotionskate-
Jugendliche mit erhöhtem Suizidrisiko zeigten Beein- trächtigungen spezifisch im Bereich der Emotionserken- nung

gorien und

Emotionsintensitäten

\section{Emotionserkennung: \\ "Faces" and "Pictures" Sub- tests (basale Emotionserken- nung) des Mayer-Solvey- \\ Emotional-Intelligence Test \\ Suizidgedanken defi- niert als Wert von mind. 1 im entsprechenden Item des Beck Depressi- ons-Inventars

\author{
keine signifikanten Unter- \\ schiede zwischen den Solda- \\ ten mit und ohne Suizidge- \\ danken bezüglich basaler \\ Emotionserkennung und ToM
}

ToM:

"Faux Pas" Geschichten Happé Strange Stories Task

\section{Emotionserkennung: \\ Suizidversuch explizit}

Erkennen der fünf Basisemotionen und neutraler Gesichter anhand von Fotos

\author{
definiert als Akt der \\ Selbstverletzung mit \\ der Intention zu sterben \\ Einschätzung als \\ "schwerwiegend" nach \\ Asberg (1976) oder an- \\ hand der Einweisung \\ auf eine Intensivstation \\ nach Überdosis \\ Pat. nach schwerwiegendem \\ Suizidversuch bezüglich des \\ Erkennens von Ekel schlech- \\ ter als die beiden anderen \\ Gruppen und tendenziell \\ schlechter bei Angst im Ver- \\ gleich zu gesunden Kontroll- \\ personen; unabhängig von \\ anderen kognitiven Dysfunk- \\ tionen
}




\begin{tabular}{llll}
\hline $\begin{array}{l}\text { Autor_innen/ Stichprobe(n) } \\
\text { Jahr }\end{array}$ & $\begin{array}{l}\text { Operationalisierung Emoti- } \\
\text { onserkennung und ToM/Em- } \\
\text { pathie }\end{array}$ & $\begin{array}{l}\text { Operationalisierung } \\
\text { Suizidalität }\end{array}$ & Zentrale Ergebnisse \\
\hline
\end{tabular}

\begin{tabular}{ll}
\hline Seymour et & 30 stationär behandelte Ju- \\
al., 2016 & gendliche nach Suizidversuch, \\
& 31 stationär behandelte Ju- \\
& gendliche mit nicht-suizidalem \\
& selbstverletzendem Verhalten \\
& und 30 gesunde Kontrollperso- \\
& nen, U.S.A., alle 13-17 Jahre \\
& alt
\end{tabular}

\section{Emotionserkennung:}

DANVA2 Emotional Face Recognition Task: glückliche, traurige, ängstliche oder wütende Gesichter in zwei unterschiedlichen Intensitätsstufen anhand von statischen Fotos von Kindern oder Erwachsenen erkennen

\author{
Erfassung von Suizidver- \\ suchen (Selbstverletzung \\ mit Absicht zu sterben) \\ und nicht-suizidalen \\ Selbstverletzungen in \\ den letzten 30 Tagen \\ Vorgeschichte nicht-sui- \\ zidaler Selbstverletzung \\ Ausschlusskriterium für \\ Gruppe nach Suizidver- \\ such/Vorgeschichte von \\ Suizidversuchen Aus- \\ schlusskriterium für die \\ Gruppe mit Selbstverlet- \\ zungen

\section{Suizidversuch explizit} \\ definiert als Akt der \\ Selbstverletzung mit \\ der Absicht zu sterben \\ Selbst- und Fremdaus- \\ mehr Fehler beim Erkennen \\ komplexer sozialer Emotio- \\ nen bei depressiven Pat. \\ nach Suizidversuch im Ver- \\ gleich zu Gesunden
}

\begin{tabular}{|c|c|}
\hline $\begin{array}{l}\text { Szanto et al., } \\
2012\end{array}$ & $\begin{array}{l}90 \text { Personen über } 60 \text { Jahre in } \\
\text { England, davon: } 24 \text { stationär } \\
\text { behandelte depressive Pat. } \\
\text { nach Suizidversuch (auch Ko- } \\
\text { morbidität mit Angststörung } \\
\text { und Substanzmissbrauch zuge- } \\
\text { lassen), } 38 \text { stationär behandelte } \\
\text { depressive Pat. ohne Suizidver- } \\
\text { such oder - gedanken aktuell } \\
\text { und in der Vorgeschichte, } 28 \\
\text { gesunde Kontrollpersonen }\end{array}$ \\
\hline $\begin{array}{l}\text { Tsypes et al., } \\
2016\end{array}$ & $\begin{array}{l}\text { Untersuchung von } 202 \text { U.S.- } \\
\text { amerikanischen Kindern (8-14 } \\
\text { Jahre) und ihren Müttern über } \\
2 \text { Jahre (alle } 6 \text { Monate), davon } \\
\text { a) Mutter depressiv/ Kind mit } \\
\text { Suizidgedanken ( } N=35) \text { b) } \\
\text { Mutter depressiv/ Kind keine } \\
\text { Suizidgedanken ( } N=76 \text { ) und c) } \\
\text { Mutter und Kind unauffällig ( } N \\
=91 \text { ) }\end{array}$ \\
\hline
\end{tabular}

\section{Emotionserkennung} Reading the Mind in the Eyes Test: Erkennung komplexer sozialer Emotionen

\section{Emotionserkennung:}

glückliche, traurige, wütende und neutrale Gesichter erkennen, die ausgehend von einem neutralen Gesicht in $10 \mathrm{er}$ Schritten gemorpht werden kunft, medizinische Unterlagen; Beck's Scale for Suicidal Ideation Suicidal Intent Scale Beck's Lethality Scale

Frage nach Suizidgedanken als Teil des Interviews „Schedule for Affective Disoders and Schizophrenia for School Age Children" (aktuell und Vorgeschichte)

Effekt verschwindet bei Kontrolle für allgemeines kognitives Funktionsniveau

Kinder mit Suizidgedanken und depressiven Müttern klassifizierten in der höchsten Intensität gemorphte wütende Gesichter als traurig

Gesamtanteil fehlgedeuteter wütender Gesichter auf den hohen Intensitätsleveln sowie spezifisch der Anteil als traurig fehlgedeuteter Gesichter sagte das Einsetzen von Suizidgedanken über einen 2-Jahres-Zeitraum voraus

Villa et al., 101 ambulante Pat. mit einer

$2018 \quad$ psychotischen Störung (74.3\% mit Schizophrenie oder einer schizoaffektiven Störung, 26\% mit einer bipolaren Störung) in den U.S.A (24-77 Jahre, $M=49.9$ Jahre)

bei $67.3 \%$ zu irgendeinem Zeitpunkt passive Suizidgedanken, 30.7\% aktive Suizidgedanken und $39.6 \%$ Suizidversuche
Emotionserkennung zwei Teilaufgaben aus der Penn Computerized Neurocognitive Battery: Differenzierung zwischen Emotionskategorien und Emotionsintensitäten

\section{Interpersonal Needs} Questionnaire: Subskalen „perceived burdensomeness“ und „thwarted belongingness“

Modified Scale for Suicidal Ideation (MSSI): Suizidgedanken innerhalb der letzten $48 \mathrm{~h}$

Columbia Suicide Severity Rating Scale: Suizidalität über die Lebenszeit bessere Emotionserkennung hing negativ mit „perceived burdensomeness“ und ,thwarted belongingness" zusammen, die Tendenz neutrale Gesichter als „ärgerlich“ fehlzuinterpretieren hingegen positiv

kein signifikanter Zusammenhang zwischen Emotionserkennung und aktuellen sowie früheren Suizidgedanken und suizidalen Verhaltensweisen

deskriptiver, aber nicht signifikanter Trend in Richtung der Fehlinterpretation neutraler Gesichter als „wütend“ bei Personen mit vorherigen Suizidgedanken/-versuchen. 


\section{Emotionserkennung}

Zehn Studien untersuchten die Fähigkeit zum Erkennen von Emotionen, alle mit Fokus auf emotionale Gesichtsausdrücke. Acht dieser Studien (Brick et al., 2019; Depp, Villa, Schembari, Harvey \& Pinkham, 2018; Ortuño-Sierra, Aritio-Solana, Del Casal \& Fonseca-Pedrero, 2020; Paradiso, Beadle, Raymont \& Grafman, 2016; Richard-Devantoy, Guillaume, Olié, Courtet \& Jollant, 2013; Seymour et al., 2016; Szanto et al., 2012; Tsypes, Burkhouse \& Gibb, 2016; Villa et al., 2018) nutzten statische Fotos von Gesichtern, welche entweder alle oder eine Auswahl der Basisemotionen (Glück, Traurigkeit, Ärger, Überraschung, Angst) darstellten, zum Teil im Vergleich mit neutralen Gesichtern. Fünf Studien (Barzilay et al., 2019; Brick et al., 2019; Depp et al., 2018; Ortuño-Sierra et al., 2020; Villa et al., 2018) verwendeten jeweils eine (Differenzierung zwischen Emotionskategorien) oder zwei (Differenzierung zwischen Emotionskategorien und -intensitäten) Teilaufgaben aus der Penn PC-gestützten Neurokognitiven Testbatterie (Gur et al., 2001). In einer der bereits genannten Studien (Depp et al., 2018) wurden zusätzlich Videodarstellungen von basalen Emotionen dargeboten, in denen Darsteller_innen die Emotionen nicht nur mimisch, sondern auch anhand der Prosodie und des Körperausdrucks zum Ausdruck brachten. Da sich die Studien hinsichtlich der mit der Suizidalität einhergehenden Ätiologien heterogen darstellen und sich gleichzeitig auf unterschiedliche Altersgruppen beziehen (Kinder/Jugendliche, Erwachsene und geriatrische Patient_innen), während hinsichtlich der Emotionserkennung in der Literatur vielfach Alterseffekte berichtet werden (Isaacowitz, Livingstone \& Castro, 2017), bot sich für diesen Abschnitt eine Darstellung der Studienergebnisse gruppiert für die verschiedenen Altersgruppen an. Kritisch ist jedoch vorab anzumerken, dass die Altersgruppen in einzelnen Studien nicht trennscharf voneinander abgegrenzt wurden.

Fünf Studien untersuchten Kinder und Jugendliche (Barzilay et al., 2019; Brick et al., 2019; Ortuño-Sierra et al., 2020; Seymour et al., 2016; Tsypes et al., 2016). Brick et al. (2019) berichteten eine genauere und schnellere Emotionsidentifikation in einer Stichprobe von Kindern und Jugendlichen im Alter von 8 bis 21 Jahren, die aufgrund verschiedener körperlicher Erkrankungen stationär behandelt worden waren und unter Suizidgedanken litten, im Vergleich zu denjenigen ohne Suizidgedanken. Diese Stichprobe wurde als Teil der sogenannten Penn Neurokognitiven Kohorte untersucht, aus der auch die Stichprobe von Barzilay et al. (2019) entstammte. Es ist unklar, inwiefern sich die Stichproben überlappten. Barzilay et al. (2019) fanden bezogen auf die Emotionserkennung jedoch keine signifikanten Unterschiede zwischen den Kindern und Jugendlichen mit und ohne Suizidgedanken, wenn die Daten zusätzlich in Abhängigkeit von Geschlecht und Pubertätsstatus (prä/post) analysiert wurden. Ortuño-Sierra et al. (2020) nutzten dieselben Aufgaben und untersuchten eine Stichprobe von Schüler_innen im Alter von 14 bis 19 Jahren aus einem zufällig ausgewählten, stratifizierten Cluster. In dieser Studie wiesen die Jugendlichen mit Suizidgedanken im Vergleich zu denjenigen ohne Suizidgedanken Beeinträchtigungen der Emotionsidentifikation auf. In einer kleineren Stichprobe von 202 Kindern ( 8 bis 14 Jahre) mit oder ohne Suizidgedanken und mit oder ohne depressive Mütter zeigte sich, dass die Kinder mit Suizidgedanken und depressiven Müttern dazu neigten, wütende Gesichter als traurig fehlzuklassifizieren (Tsypes et al., 2016). Eine entsprechende Fehlklassifikation erwies sich überdies als prädiktiv für das Einsetzen von Suizidgedanken im 2-jährigen Untersuchungszeitraum. Der Zusammenhang zwischen Emotionserkennung und suizidalem Erleben/Verhalten mag also durch verschiedene Faktoren (z.B. genetische Prädispositionen und/oder das Zusammenleben mit einem depressiven Elternteil) moderiert werden. Eine letzte Studie untersuchte schließlich psychiatrisch hospitalisierte Jugendliche, die in den vergangenen 30 Tagen entweder einen Suizidversuch unternommen oder aber nichtsuizidales selbstverletzendes Verhalten gezeigt hatten (Seymour et al., 2016). Hier wiesen die Jugendlichen nach Suizidversuch im Vergleich zu gesunden Kontrollpersonen vergleichbare Leistungen in der Emotionserkennung auf. Lediglich die Jugendlichen mit nichtsuizidalem selbstverletzendem Verhalten zeigten Beeinträchtigungen in der Emotionserkennung im Vergleich zu den Kontrollpersonen.

Vier Studien fokussierten auf erwachsene Patient_innen im mittleren Altersbereich (Depp et al., 2018; Paradiso et al., 2016; Richard-Devantoy et al., 2013; Villa et al., 2018). Depp et al. (2018) und Villa et al. (2018) untersuchten jeweils ambulant behandelte Patient_innen mit psychotischen Erkrankungen. Die Ergebnisse gingen in beiden Studien in eine ähnliche Richtung: Bei Depp et al. (2018) fanden sich keine signifikanten Unterschiede zwischen Patient_innen mit und ohne Suizidgedanken bezüglich der Emotionserkennung anhand statischer Stimuli (Aufgabe aus der Penn-Testbatterie) und auch bei Villa et al. (2018) hing die Emotionserkennung in der gleichen Aufgabe nicht mit aktuellen und vergangenen Suizidgedanken oder suizidalen Verhaltensweisen zusammen. Allerdings waren bei Depp et al. (2018) Patient_innen mit aktuellen Suizidgedanken beim Erkennen negativer Emotionen anhand dynamischer Stimuli (Videos von Personen incl. Oberkörper und Stimme) genauer als Patient innen ohne Suizidgedanken. Die Fähigkeit zum Erkennen negativer dynamischer Stimuli sagte jedoch nicht das Auftreten von Suizidgedanken 2 Wochen später voraus. Villa et al. (2018) erhoben fragebogenbasiert zusätz- 
lich die in der Einleitung vorgestellten Konstrukte der Interpersonalen Theorie suizidalen Verhaltens und konnten zeigen, dass eine genauere Emotionserkennung (anhand von dynamischen Stimuli) mit reduziertem Lasterleben (perceived burdensomeness) und vermehrtem Zugehörigkeitserleben (thwarted belongingness) assoziiert war. Die Tendenz, neutrale Gesichter als ,ärgerlich“ fehlzuinterpretieren, war zudem positiv mit diesen Konstrukten assoziiert. Dazu passend ergab sich ein deskriptiver, aber nicht signifikanter Trend, wonach Personen mit Suizidversuchen in der Vorgeschichte auch eine verstärkte Tendenz zeigten, neutrale Gesichter als wütend fehlzuinterpretieren. Solche Fehlinterpretationseffekte bei Menschen mit einem vorangegangenen Suizidversuch zeigten sich auch bei Richard-Devantoy et al. (2013): Aktuell depressive Personen mit mindestens einem Suizidversuch in der Vorgeschichte waren schlechter dazu in der Lage, die Emotion Ekel zu erkennen, als depressive Menschen ohne vorausgegangenen Suizidversuch und gesunde Kontrollpersonen. Auch konnten sie die Emotion "Angst" schlechter erkennen als die gesunden Kontrollpersonen. Dieses Ergebnis passt zu den beschriebenen Befunden von Tsypes et al. (2016), die Fehlklassifikationseffekte bei Kindern mit Suizidgedanken und depressiven Müttern ausmachen konnten.

Paradiso et al. (2016) fanden in einer weiteren Untersuchung keine Unterschiede bezüglich der Emotionserkennung zwischen Vietnamveteranen mit und ohne Suizidgedanken. Die Studie wies jedoch eine Reihe von methodischen Problemen auf (u.a. hoher Anteil hirngeschädigter Probanden, von denen nicht berichtet wird, wie sie sich auf die beiden Gruppen suizidaler und nichtsuizidaler Teilnehmer verteilten, Gruppenunterschiede in soziodemografischen Variablen); entsprechend ist unklar, inwieweit das Befundmuster als valide erachtet werden kann (siehe hierzu z. B. Azouvi, Arnould, Dromer \& VallatAzouvi, 2017).

Szanto et al. (2012) verwendeten den „Reading the Mind in the Eyes"-Test (Baron-Cohen et al., 2001) bei 90 älteren Personen (Alter $\geq 60$ Jahre) als Test zur Erfassung komplexer sozialer Emotionen. Ebenso wie bei den Jugendlichen, die von Seymour et al. (2016) untersucht worden waren, zeigten sich depressive Patient_innen nach Suizidversuch nicht aber solche ohne Suizidversuch oder -gedanken in der Vorgeschichte im Vergleich zu gesunden Kontrollpersonen in der Emotionserkennung beeinträchtigt. Dieser Effekt verschwand jedoch bei Kontrolle des allgemeinen kognitiven Funktionsniveaus. Angemerkt sei des Weiteren, dass der "Reading the Mind in the Eyes“Test aufgrund der komplexen Aufgabenstellung von vielen Autor_innen (z. B. Gallant \& Good, 2020) eher als Aufgabe zur Erfassung der kognitiven Empathie/affektiven ToM verstanden wird.
Zusammenfassend findet sich ein sehr heterogenes Befundmuster bezüglich des Zusammenhangs zwischen der Emotionserkennung und suizidalem Erleben/Verhalten. In zwei Studien wurden in Zusammenhang mit Suizidgedanken und/oder Suizidversuch bessere Leistungen (Brick et al., 2019; Depp et al., 2018), in fünf Studien schlechtere Leistungen (Ortuño-Sierra et al., 2020; Richard-Devantoy et al., 2013; Szanto et al., 2012; Tsypes et al., 2016; Villa et al., 2018) und in vier Studien keine Unterschiede (Barzilay et al., 2019; Depp et al., 2018; Paradiso et al., 2016; Seymour et al., 2016) berichtet. Es zeichnet sich ab, dass unter Umständen emotionsspezifische Effekte bestehen, wonach sich insbesondere für angeekelte Gesichtsausdrücke Beeinträchtigungen zeigen (Richard-Devantoy et al., 2013) und Fehlklassifikationen von neutralen Gesichtern als wütend (Villa et al., 2018) und wütenden Gesichtern als traurig möglicherweise einen Vulnerabilitätsmarker für Suizidversuche darstellen (Tsypes et al., 2016).

\section{Empathie}

Es konnte anhand der für die Literaturrecherche vordefinierten Kriterien nur eine Studie (Harenski et al., 2017) identifiziert werden, in der Empathie direkt und mit Verhaltens-/Fähigkeitsmaßen und nicht mithilfe von Selbstberichtsmaßen untersucht wurde. Harenski et al. (2017) verwendeten Videos, in denen reale Protagonist_innen (keine Schauspieler_innen) von ihren emotionalen Erlebnissen erzählen. Die untersuchten Personen wählten aus einer Liste diejenigen Emotionen aus, von denen sie glauben, dass die Hauptperson sie erlebt hat, und ordneten diese nach Relevanz. Die „empathische Genauigkeit“ wurde als Grad der Übereinstimmung mit den tatsächlich von den Protagonist_innen angegebenen Emotionen bestimmt. Die von Harenski et al. (2017) untersuchten männlichen Forensikpatienten mit psychotischen Erkrankungen und Suizidversuchen in der Vorgeschichte zeigten eine verminderte empathische Genauigkeit im Vergleich zu den psychotischen Forensikpatienten ohne lebenszeitliche Suizidversuche sowie im Vergleich zu den nichtpsychotischen Straffälligen und gesunden Kontrollpersonen. Eine verminderte empathische Genauigkeit war dabei mit einem erhöhten Risiko für vergangene Suizidversuche assoziiert, unabhängig von anderen Risikofaktoren. In dieser Studie wurden die Probanden auch im Kernspintomographen untersucht, was für die betroffene Subgruppe von Patienten parallel auch eine Volumenreduktion der grauen Substanz in Arealen (bilateral in den Temporalpolen), die wichtig für die soziokognitive Verarbeitung sind, aufzeigte.

Zusammenfassend verweisen die Ergebnisse der einzigen Studie zum Themenbereich übereinstimmend auf 
eine verminderte Empathiefähigkeit im Kontext suizidalen Erlebens und Verhaltens.

\section{Theory of Mind (ToM)}

In fünf Publikationen (zwei beziehen sich auf dieselbe Studie: Canal-Rivero et al., 2017, 2019) wurde ToM in Zusammenhang mit suizidalem Erleben und Verhalten untersucht. Abgesehen von einer Untersuchung, in der ToM filmbasiert erfasst wurde (Hatkevich, Venta \& Sharp, 2019), kamen dabei überwiegend schriftlich formulierte Fallvignetten zum Einsatz (Canal-Rivero et al., 2017, 2019; Duñó et al., 2009; Paradiso et al., 2016), in denen die Proband_innen den mentalen Zustand der Hauptperson (ToM-Aufgabe erster Ordnung) oder die Annahmen der Hauptperson über den mentalen Status einer weiteren Person (ToM-Aufgabe zweiter Ordnung) erschließen mussten. Duñó et al. (2009) untersuchten remittierte Patient_innen mit Schizophrenie und konnten zeigen, dass Patient innen mit lebenszeitlichen Suizidversuchen Beeinträchtigungen im Verständnis von ToM-Geschichten zweiter Ordnung aufwiesen. Diese ToM-Beeinträchtigungen zweiter Ordnung erwiesen sich überdies als signifikante Prädiktoren für lebenszeitliche Suizidversuche - und dies auch dann, wenn der Intelligenzquotient kontrolliert wurde, nicht aber wenn eine schlechtere psychosoziale Anpassung in der Kindheit berücksichtigt wurde. Paradiso et al. (2016) fanden in ihrer Untersuchung männlicher Vietnamveteranen mit oder ohne akute Suizidgedanken, dass sich die beiden Subgruppen nicht signifikant hinsichtlich der Leistungen in den verwendeten ToM-Aufgaben (Interpretation von Fauxpas und klassischen ToM-Geschichten) unterschieden. Diese Befunde sind jedoch aufgrund der bereits beschriebenen methodischen Probleme (u.U. Einschluss hirngeschädigter Patient_innen in beiden Gruppen) nur schwer valide interpretierbar.

Canal-Rivero et al berichten in zwei Publikationen $(2017,2019)$ über eine Stichprobe überwiegend junger Erwachsener, die aufgrund einer ersten psychotischen Episode stationär psychiatrisch behandelt und über einen 12-monatigen Zeitraum nachuntersucht wurden. Erhoben wurde, inwiefern sich Patient_innen, die innerhalb dieses Zeitraums mindestens einen Suizidversuch unternahmen, hinsichtlich ihrer ToM-Fähigkeiten von denen unterschieden, die keinen Suizidversuch unternommen hatten (Canal-Rivero et al., 2017). Dabei zeigten Proband_innen mit Suizidversuch signifikant mehr ToM-Fehler erster und zweiter Ordnung als Proband_innen ohne Suizidversuch. Lediglich die ToM-Fehler erster Ordnung erwiesen sich jedoch als unabhängige Prädiktoren von Suizidversuche im Nachuntersuchungszeitraum. In einer zweiten Publikation berichten Canal-Rivero et al. (2019) von einer Zusatzana- lyse, die sich auf die Anzahl der Suizidversuche im Nachuntersuchungszeitraum bezog. So unternahmen sowohl Patient_innen, die in ToM-Geschichten erster Ordnung, als auch solche, die in ToM-Geschichten zweiter Ordnung fehlerhafte Antworten gaben, mehr Suizidversuche als diejenigen, die jeweils fehlerfrei antworteten. Erneut sagte dabei jedoch nur die Anzahl der Fehler in ToM-Geschichten erster, nicht aber zweiter Ordnung die Anzahl der Suizidversuche voraus. Die Diskrepanz zu den Befunden von Duñó et al. (2009) erklären Canal-Rivero et al. (2017) mit möglichen zustandsabhängigen Effekten. So mag es sein, dass in akuteren Erkrankungsphasen, wie bei Canal-Rivero et al. (2017, 2019), schon ToM-Beeinträchtigungen ersten Grades die Wahrscheinlichkeit eines Suizidversuchs erhöhen. In stabileren Phasen der Remission, wie bei Duñó et al. (2009), könnten hingegen Beeinträchtigungen der Fähigkeit, komplexere Schlussfolgerungen über die mentalen Attributionen anderer Personen bezogen auf Dritte zu ziehen, zum Tragen kommen. Zusätzlich ist zu berücksichtigen, dass bei Duñó et al. (2009) eine retrospektive Einschätzung der Suizidalität erfolgte, während Canal-Rivero et al. $(2017,2019)$ eine Verlaufsuntersuchung über 12 Monate durchführten.

Hatkevich et al. (2019) haben in ihrer Untersuchung einen weiteren interessanten Aspekt ins Spiel gebracht. Sie erfassten ToM filmbasiert mithilfe des „Movie for the Assessment of Social Cognition“" (Dziobek et al., 2006). Dabei wurden den Proband_innen im Multiple-Choice-Format ToM-Fragen zu den gezeigten sozialen Interaktionssituationen gestellt und auf Basis der Antworten vier Indizes abgeleitet: keine ToM, weniger ToM, akkurate ToM oder ,exzessive ToM". Letztere meinte das übertriebene Zuschreiben von mentalistischen Zuständen, z. B. wenn weit hergeholte Erklärungen für das (neutrale) Verhalten anderer Personen herangezogen wurden. Sie untersuchten eine größere Stichprobe aufgrund verschiedener psychiatrischer Diagnosen hospitalisierter Jugendlicher im Alter von 12 bis 17 Jahren und fanden, dass spezifisch die Neigung zum Übermentalisieren (,exzessive ToM“), nicht aber andere Arten der inkorrekten ToM-Antworten, mit Suizidgedanken in den letzten 4 Wochen und Suizidversuchen im letzten Jahr positiv zusammenhing. Exzessive ToM erwies sich als ein von depressiver Symptomatik unabhängiger Prädiktor, der auch signifikant blieb, wenn für verschiedene demografische Faktoren wie Alter und Geschlecht kontrolliert wurde. Die Autor_innen gingen davon aus, dass die Neigung zum Überinterpretieren von sozialen Informationen, die in der exzessiven ToM-Antworttendenz zum Ausdruck kommen könnte, in der Alltagskommunikation maladaptiv ist und $\mathrm{zu}$ hypersensitiven Reaktionen spezifisch auf wahrgenommene negative soziale Reize führen könnte. Sie stellten eine Verbindung zu Joiners (2005) Interpersonaler Theorie suizidalen Verhaltens her, indem sie postulierten, dass die 
Überinterpretationen in negativer Richtung auch damit assoziiert sein könnten, dass die Jugendlichen dazu neigen, sich fälschlicherweise als Bürde für Interaktionspersonen wahrzunehmen und weniger Zugehörigkeitsgefühl zu erleben.

Zusammenfassend legen die bisherigen wenigen Studien überwiegend nahe, dass Veränderungen der ToM-Fähigkeiten, sowohl im Sinne von fehlerhaft attribuierten mentalen und emotionalen Zuständen als auch in Form übertriebener Zuschreibungen von mentalen Zuständen, mit erhöhter Suizidalität, primär in Form von manifesten Suizidversuchen, einhergehen können.

\section{Diskussion}

Insgesamt konnten 15 Artikel zum Zusammenhang von Emotionserkennung, Empathie und ToM auf der einen Seite und suizidalem Erleben und Verhalten auf der anderen Seite anhand der vordefinierten Suchkriterien identifiziert werden. Auffällig ist zunächst, dass sich die Literatur erst in den letzten 10 Jahren eingehender mit diesen $\mathrm{Zu}$ sammenhängen beschäftigt zu haben scheint. Die Befundlage stellt sich dabei heterogen dar, insbesondere für den Bereich der Emotionserkennung mit zehn Studien, die sowohl verbesserte, beeinträchtigte als auch unauffällige Fähigkeiten zur Erkennung emotionaler Ausdrücke anhand von Gesichtern berichten. Für den Bereich der Empathie wurde nur eine Studie identifiziert, die eine verminderte Empathiefähigkeit in Zusammenhang mit suizidalem Erleben und Verhalten nahelegte. Bezüglich der ToM (fünf Publikationen) schienen sowohl Defizite als auch eine Tendenz zum übergenauen oder exzessiven Attribuieren von mentalen Zuständen in Zusammenhang mit suizidalem Erleben und Verhalten zu stehen.

Eine verbesserte Emotionserkennung kann in Bezug auf Suizidalität protektiv wirken, möglicherweise weil Emotionen wichtige soziale Signale senden, deren korrekte Interpretation maßgeblich zum Gelingen sozialer Interaktionen beiträgt. So konnten beispielsweise Villa et al. (2018) einen Zusammenhang zwischen besserer Emotionserkennung und vermindertem Lasterleben (perceived burdensomeness) sowie intensiver erlebter sozialer Zugehörigkeit (belongingness) zeigen. Die in einigen Studien festgestellten Beeinträchtigungen der Emotionserkennung in $\mathrm{Zu}-$ sammenhang mit verstärktem suizidalen Erleben und Verhalten scheinen teilweise emotionsspezifische Effekte zu reflektieren, im Sinne von Fehlleistungen spezifisch für angeekelte Gesichter (Richard-Devantoy et al., 2013) bzw. Fehlklassifikationen von insbesondere wütenden Gesichtern als traurig (Tsypes et al., 2016). Richard-Devantoy et al. (2013) vermuteten einen Zusammenhang mit einer möglicherweise verminderten Funktionsweise der Insula und des orbitofrontalen Kortex. Diese Strukturen sind nicht nur eng miteinander vernetzt, sondern auch beide mit der Verarbeitung sozial aversiver Stimuli betraut (Adolphs, 2001) und weisen bei Personen mit suizidalen Verhaltensweisen und Gedanken funktionelle und strukturelle Auffälligkeiten auf (Auerbach et al., 2021).

Auf der anderen Seite kann sich die Tendenz, überdurchschnittliche gute Leistungen in der Emotionserkennung zu zeigen (Brick et al., 2019; Depp et al., 2018) sowie mentale Zustände anderer zu überinterpretieren, auch maladaptiv auswirken und mit erhöhter Suizidalität assoziiert sein (Hatkevich et al., 2019). Dies passt zu Befunden von Wang et al. (2020), die für Patient_innen mit Schizophrenie und lebenszeitlichen Suizidversuchen im Vergleich zu Patient_innen ohne Suizidversuche im Selbstbericht eine erhöhte persönliche Betroffenheit im Sinne einer affektiven Empathiekomponente nachweisen konnten. Dieser vermutlich zunächst ungewöhnlich klingende Zusammenhang wurde auch in einigen früheren Studien schon für das allgemeine kognitive Funktionsniveau gefunden, wobei bessere kognitive Funktionen hier mit erhöhter Suizidalität einhergingen, was damit erklärt werden könnte, dass die Planung und Umsetzung eines Suizids entsprechende kognitive Leistungen, z. B. im Bereich der exekutiven Funktionen, erfordert (z.B. Kim et al., 2003; Villa et al., 2018). Die oben zitierten Befunde von Wang et al. (2020) stützen die naheliegende Annahme, dass in diesem Zusammenhang möglicherweise v.a. eine mit den besseren Leistungen im Bereich der Emotionserkennung/Empathie einhergehende stärkere persönliche emotionale Reaktion problematisch sein könnte. So war bei Depp et al. (2018) auch v.a. die bessere Erkennung negativer Emotionen in dynamischen (und somit naturalistischeren) Darstellungen emotionaler Stimuli mit erhöhter Suizidalität assoziiert. Diese Ergebnisse legen die Schlussfolgerung nahe, dass nicht nur eine Verbesserung von Verhaltensfertigkeiten im Bereich der sozialen Kognitionen einen wichtigen therapeutischen Ansatzpunkt darstellen könnte, sondern im Einzelfall auch die Vermittlung eines adäquaten Umgangs - z. B. im Sinne der Emotionsregulation mit übersteigerten soziokognitiven Fähigkeiten.

Grundsätzlich weisen die bisherigen Studien eine erhebliche Variabilität bezüglich verschiedener methodischer Aspekte auf. So wurden sie in verschiedenen Ländern durchgeführt (Spanien, USA, Mexiko), beziehen sich auf unterschiedliche Altersgruppen (Kinder/Jugendliche, Erwachsene, geriatrische Patient_innen), Erkrankungen (Psychosen, heterogene psychiatrische Stichproben, aber auch Personen ohne relevante psychische Erkrankungen) und Erkrankungsphasen (akut hospitalisiert bis hin zu ambulant remittiert). 
Die in den Studien untersuchten Altersgruppen waren nicht klar voneinander abgegrenzt, z. B. wurden teilweise Kinder und Jugendliche im Alter bis zu 21 Jahren untersucht (Barzilay et al., 2019; Brick et al., 2019). Bei diesen Gruppen liegt die besondere Bedeutung der frühen Identifikation potenzieller Vulnerabilitätsmarker für suizidales Erleben und Verhalten auf der Hand, z. B. im Hinblick auf die mögliche Entwicklung von präventiven Ansätzen wie beispielsweise der Förderung von Kompetenzen der Emotionserkennung (im Falle beeinträchtigter Leistungen) und der Emotionsregulation (im Falle einer gesteigerten Fähigkeit, subtile emotionale Signale wahrzunehmen). In der Untersuchung von Barzilay et al. (2019) konnten zwar bezüglich der Emotionserkennung keine relevanten Effekte von Alter und Geschlecht gefunden werden, es zeigte sich jedoch, dass das allgemeine kognitive Funktionsniveau v.a. bei männlichen Jugendlichen nach der Pubertät erhöht war, wenn diese Suizidgedanken hegten, im Vergleich zu postpubertären Jugendlichen ohne Suizidgedanken. In der Studie von Tsypes et al. (2016) sagten Fehlklassifikationen von wütenden Gesichtern als traurig Suizidversuche 2 Jahre später voraus. Da es sich um Kinder depressiver Mütter handelte, könnten die beschriebenen Fehlklassifikationen möglicherweise neben einer möglichen genetischen Vulnerabilität (für eine Übersicht siehe Vaquero-Lorenzo \& Vasquez, 2020) einen zusätzlichen Prädiktor für suizidales Erleben und Verhalten darstellen. Wenn auch die Studien sich bezüglich der verwendeten Testverfahren zur Erfassung sozialer Kognitionen als relativ homogen erweisen was wohl auch der vergleichsweise überschaubaren Anzahl von zur Verfügung stehender Testverfahren geschuldet ist -, so variiert die Erfassung suizidalen Erlebens und Verhaltens erheblich. Während teilweise die Einschätzung der Suizidalität nur anhand eines einzelnen Suiziditems erfolgt (z.B. Barzilay et al., 2019; Depp et al., 2018; Paradiso et al., 2016), werden in anderen Studien mehrere Datenquellen und aufwendige Skalen herangezogen, welche nicht nur Suizidgedanken, sondern auch die Suizidversuche und deren Schweregrad erheben (z.B. Szanto et al., 2012). Allerdings wurde dabei auch nicht immer akkurat zwischen suizidalen und nichtsuizidalen Selbstverletzungen differenziert (Canal-Rivero et al., 2017, 2019), obwohl diese Unterscheidung relevant ist (Posner, Brodsky, Yershova, Buchanan \& Mann, 2014), wie auch anhand der Befunde von Seymour et al. (2016) ersichtlich wird, die eine beeinträchtigte Emotionserkennung im Vergleich zu Kontrollpersonen nur für Jugendliche mit nichtsuizidalem selbstverletzenden Verhalten, nicht aber für Jugendliche mit suizidalem selbstverletzenden Verhalten zeigen konnten. Zudem wurden nicht in allen Studien Suizidgedanken und Suizidversuche erhoben bzw. differenziert. Darüber hinaus sollten weitere konfundierende Va- riablen, wie das allgemeine kognitive Funktionsniveau, berücksichtigt werden, da z. B. bei Szanto et al. (2012) signifikante Effekte für Beeinträchtigungen im Bereich sozialer Kognitionen nach Kontrolle dieser Variablen wieder verschwunden sind. In anderen Studien blieben die berichteten Effekte für den Zusammenhang zwischen Suizidalität und sozialen Kognitionen jedoch stabil, wenn für kognitive Funktionen kontrolliert wurde (Richard-Devantoy et al., 2013; Villa et al., 2018). Wie in der Einleitung bereits dargelegt wurde, wäre insbesondere die Kontrolle von zentralen Aspekten exekutiver Funktionen essenziell (Channon, 2004); diese wurde jedoch nicht in allen Studien berücksichtigt. Außerdem ist zu bedenken, dass eine psychotrope Medikation die neuronalen Verarbeitungsprozesse beeinflussen kann, die sozialen Kognitionen zugrunde liegen. Beispielsweise wurde gezeigt, dass die Behandlung mit Antidepressiva bei depressiven Menschen überschießende Reaktionen der Amygdala auf ängstliche Gesichter verringern kann (z.B. Arnone et al., 2012). Passend dazu berichten Seymour et al. (2016) in ihrer in dieser Übersichtsarbeit eingeschlossenen Studie, dass sich anhand der statistischen Kontrolle für die Einnahme von Selektiven Serotonin-Wiederaufnahmehemmern zeigen ließ, dass diese möglicherweise bestehende Defizite der Gesichterverarbeitung bei depressiven Jugendlichen verringern. Allerdings galt dies spezifisch für Jugendliche, die lediglich nichtsuizidale selbstverletzende Verhaltensweisen zeigten. Im Gegensatz dazu scheinen Antipsychotika möglicherweise soziokognitive Funktionen eher zu beeinträchtigen (Monteleone et al., 2021). Harenski et al. (2017) fanden in ihrer Studie jedoch keine Zusammenhänge zwischen empathischer Genauigkeit und der eingenommenen antipsychotischen Medikation, die außerdem über alle klinischen Gruppen vergleichbar war. Auch Tsypes et al. (2016) berichteten über robuste Effekte, wenn für psychiatrische Medikation kontrolliert wurde. Leider wurde in den meisten Studien der vorliegenden Übersicht nicht bezüglich der Auswirkungen von entsprechender Medikation explizit auf das soziokognitive Funktionsniveau kontrolliert, auch wenn in einigen Fällen berichtet wurde, dass die Verteilung der eingesetzten Substanzklassen zumindest über die verschiedenen klinischen Subgruppen vergleichbar war (Szanto et al., 2012).

Die Literaturrecherche für die vorliegende Übersichtsarbeit wurde unabhängig von spezifischen Ätiologien durchgeführt, vor deren Hintergrund Suizidalität eine Rolle spielen könnte. Vorwiegend wurden in den eingeschlossenen Untersuchungen Patient_innen mit psychotischen Erkrankungen, v. a. aus dem schizophrenen Formenkreis, untersucht (Canal-Rivero et al., 2017, 2019; Depp et al., 2018; Duñó et al., 2019; Harenski et al., 2017; Villa et al., 2018). Drei Studien fokussierten auf Personen 
mit depressiven Erkrankungen, entweder aktuell (Szanto et al., 2012), in der Vorgeschichte (Richard-Devantoy et al., 2013) oder als Angehörige depressiver Mütter (Tsypes et al., 2016). Die sechs Studien mit stark heterogenen Stichproben (z.T. Stichprobe nur körperlich vorerkrankter Jugendlicher, z.T. zufällig stratifiziertes Cluster aus einer Gesamtstichprobe von Schüler_innen) beziehen sich v. a. auf Kinder und Jugendliche (Barzilay et al., 2019; Brick et al., 2019; Hatkevich et al., 2019; Ortuño-Sierra et al., 2020; Seymour et al., 2016) bzw. eine retrospektive Analyse von Vietnamveteranen (Paradiso et al., 2016). Bei den psychotischen Patient_innen scheint recht einheitlich eine Beeinträchtigung komplexerer sozialer Kognition in Form einer defizitären ToM (Canal-Rivero et al., 2017, 2019; Duñó et al., 2019) und einer verminderten empathischen Genauigkeit (Harenski et al., 2017) mit erhöhter Suizidalität zusammenzuhängen. Für die Emotionserkennung zeigt sich hingegen ein diskrepantes Bild: Während Depp et al. (2018) eine genauere Erkennung von dynamisch dargestellten negativen Emotionen mit vermehrten aktuellen Suizidgedanken assoziierten, so war dies in einer im gleichen Jahr veröffentlichten Untersuchung von Villa et al. (2018) an einer ansonsten vergleichbaren (Alter, Diagnose, Behandlungsstatus) Stichprobe nicht der Fall. Im Gegenteil, die verbesserte Emotionserkennung hing hier sogar mit dem Empfinden eines verminderten Lasterlebens (perceived burdensomeness) und einer erhöhten sozialen Zugehörigkeit (belongingness) zusammen, was insgesamt als Erklärung für einen Zusammenhang zwischen besseren soziokognitiven Leistungen und verminderten Suizidalität einleuchtet. Möglicherweise spielt hier jedoch eine Rolle, dass ein Viertel der Patient_innen in dieser Studie eine bipolare Erkrankung hatte; andererseits zeigte sich auch bei Hatkevich et al. (2019) bei Jugendlichen mit überwiegend affektiven Störungen, dass eine übertriebene Zuschreibung von mentalen Zuständen (eher) positiv mit Suizidgedanken zusammenhing. Bei Patient_innen mit einer Vulnerabilität für depressive Erkrankungen, entweder aufgrund eigener positiver Vorgeschichte (Richard-Devantoy et al., 2013) oder einer positiven Familienanamnese (Tsypes et al., 2016), scheinen v. a. Schwierigkeiten bei der Interpretation der Emotionen Ekel und Wut zu bestehen.

Die Literatursuche erbrachte keinen einzigen Treffer für eine Studie, die den Zusammenhang zwischen Suizidalität und sozialen Kognitionen nach den vordefinierten Kriterien explizit bei Patient innen mit erworbenen Hirnschädigungen beleuchtet. In zwei Untersuchungen fand dieser Aspekt jedoch partielle Berücksichtigung: Bei Szanto et al. (2012) waren die Einschränkungen im Bereich der kognitiven Empathie nach Suizidversuch nicht korreliert mit dem Auftreten von hypoxischen Hirnschädigungen infolge der Suizidversuche. Allerdings war im
Rahmen dieser Studie nicht zu eruieren, inwiefern die für soziokognitive Funktionen relevanten fronto-temporalen Schaltkreise von den Hirnschädigungen betroffen waren. Die Stichproben von Paradiso et al. (2016) umfassten auch hirngeschädigte Patient_innen, die jedoch unsystematisch auf die verschiedenen Gruppen suizidaler und nichtsuizidaler Patient_innen verteilt waren, sodass keine validen Schlussfolgerungen spezifisch für diese Patient_innengruppe gezogen werden konnten. Wie in der Studie von Szanto et al. (2012) ersichtlich wird, können Hirnschädigungen nicht nur infolge eines Suizidversuchs entstehen, sondern die Suizidrate zeigt sich bei Patient innen mit erworbenen Hirnschädigungen allgemein im Vergleich zu Personen ohne neurologische Erkrankung erhöht (Erlangsen et al., 2020), insbesondere bei Vorliegen kognitiver Beeinträchtigungen (Arciniegas \& Anderson, 2002). Da inzwischen zahlreiche Untersuchungen Einschränkungen im Bereich sozialer Kognitionen bei hirngeschädigten Patient_innen nachgewiesen haben (siehe z. B. Azouvi et al., 2017), ist davon auszugehen, dass darin - gerade auch in Interaktion mit den oft ohnehin vorliegenden kognitiven Beeinträchtigungen ein weiterer potenzieller Risikofaktor für suizidales Erleben und Verhalten liegt, den es therapeutisch zu berücksichtigen gilt.

Künftige Untersuchungen sollten die oben skizzierten verschiedenen potenziellen Einflussfaktoren auf den $\mathrm{Zu}$ sammenhang zwischen sozialen Kognitionen und Suizidalität genauer ergründen. Insbesondere ist eine differenzierte Erfassung sozialer Kognitionen bezüglich der zentralen Subkonstrukte bei suizidgefährdeten Patient innen, getrennt nach ätiologischen Gesichtspunkten und Altersgruppen, wünschenswert. Ein besseres Verständnis der Bedingungen, unter denen z. B. eine eher gesteigerte Fähigkeit zur Emotionserkennung oder ToM zu einem erhöhten Suizidrisiko führt, würde zudem ermöglichen, die Behandlung zielgerichteter auf das erhobene Fähigkeitsmuster zuzuschneiden. So würde sich bei Beeinträchtigungen soziokognitiver Fähigkeiten im Sinne einer verminderten Emotionserkennung oder aber empathischer Genauigkeit ein entsprechendes Training dieser Fertigkeiten anbieten. Im Falle einer besonders geschärften Fähigkeit zur Emotionserkennung oder übersteigerter ToM sollten eher Emotionsregulationsfähigkeiten geübt werden, um ggf. übersteigerte eigene persönliche Betroffenheit adaptiv regulieren zu können. In den entsprechenden Studien sollten darüber hinaus Effekte der Medikation und des allgemeinen kognitiven Funktionsstatus berücksichtigt werden. Dennoch zeigt das (wenn auch heterogene) Befundmuster jetzt schon auf, dass Beeinträchtigungen sozialer Kognitionen in der Behandlung potenziell suizidgefährdeter Patient_innen therapeutisch berücksichtigt werden sollten, wie dies bereits in einigen Pro- 
grammen (z. B. Lindenmayer et al., 2013; Penn, Roberts, Combs \& Sterne, 2007) erfolgt, ohne dass diese spezifisch im Vorfeld für die Behandlung suizidalen Erlebens und Verhaltens konzipiert wurden.

\section{Literatur}

Adolphs, R. (2001). The neurobiology of social cognition. Current Opinion in Neurobiology, 11, 231-239. https://doi.org/10.1016/ s0959-4388 (00)00202-6

Appleby, L., Cooper, J., Amos, T. \& Faragher, B. (1999). Psychological autopsy study of suicides by people aged under 35. British Journal of Psychiatry, 175, 168-174. https://doi.org/10.1192/ bjp.175.2.168

Arciniegas, D. B. \& Anderson, C.A. (2002). Suicide in neurologic illness. Current Treatment Options in Neurology, 4, 457-468. https://doi.org/10.1007/s11940-002-0013-5

Arnone, D., McKie, S., Elliott, R., Thomas, E.J., Downey, D., Juhasz, G. et al. (2012). Increased amygdala responses to sad but not fearful faces in major depression: Relation to mood state and pharmacological treatment. American Journal of Psychiatry, 169, 841-850. https://doi.org/10.1176/appi.ajp.2012.11121774

Auerbach, R.P., Pagliaccio, D., Allison, G. O., Alqueza, K. L. \& Alonso, M.F. (2021). Neural correlates associated with suicide and nonsuicidal self-injury in youth. Biological Psychiatry, 89, 119-133. https://doi.org/10.1016/j.biopsych.2020.06.002

Azouvi, P., Arnould, A., Dromer, E. \& Vallat-Azouvi, C. (2017). Neuropsychology of traumatic brain injury: An expert overview. Revue Neurologique, 173, 461-472. https://doi.org/10.1016/j.neurol.2017.07.006

Baron-Cohen, S., Wheelwright, S., Hill, J., Raste, Y. \& Plumb, I. (2001). The „Reading the Mind in the Eyes“Test revised version: A study with normal adults, and adults with Asperger syndrome or high-functioning autism. Journal of Child Psychology and Psychiatry, and Allied Disciplines, 42, 241-251.

Barzilay, R., Calkins, M.E., Moore, T. M., Boyd, R.C., Jones, J. D., Benton, T.D. et al. (2019). Neurocognitive functioning in community youth with suicidal ideation: Gender and pubertal effects. British Journal of Psychiatry, 215, 552-558. https://doi.org/10.1 192/bjp.2019.55

Beautrais, A. L. (2002). A case control study of suicide and attempted suicide in older adults. Suicide \& life-threatening behavior, 32,1-9. https://doi.org/10.1521/suli.32.1.1.22184

Beer, J.S. \& Ochsner, K.N. (2006). Social cognition: A multi level analysis. Brain Research, 1079, 98-105. https://doi.org/10.10 16/j.brainres.2006.01.002

Breitborde, N., Wastler, H., Pine, J. G. \& Moe, A. M. (2020). Suicidality and social problem-solving skills among individuals with firstepisode psychosis participating in Coordinated Specialty Care. Early Intervention in Psychiatry. https://doi.org/10.1111/eip.1 2967

Brick, L.A., Marraccini, M.E., Micalizzi, L., Benca-Bachman, C.E., Knopik, V.S. \& Palmer, R. (2019). Overlapping genetic effects between suicidal ideation and neurocognitive functioning. Journal of Affective Disorders, 249, 104-111. https://doi.org/10. 1016/j.jad.2019.02.003

Brüne, M. (2005). „Theory of mind“ in schizophrenia: A review of the literature. Schizophrenia Bulletin, 31, 21-42. https://doi. org/10.1093/schbul/sbi002

Canal-Rivero, M., Lopez-Moriñigo, J.D., Barrigón, M.L., PeronaGarcelán, S., Jimenez-Casado, C., David, A.S. et al. (2017). The role of premorbid personality and social cognition in suicidal behaviour in first-episode psychosis: A one-year follow-up study. Psychiatry Research, 256, 13-20. https://doi.org/10.1016/j. psychres.2017.05.050

Canal-Rivero, M., Velasco-Barbancho, E., Barrigón, M.L., VillarCabeza, F., Obiols-Llandrich, J.E., Crespo-Facorro, B. et al. (2019). Personality traits, theory of mind and their relationship with multiple suicide attempts in a sample of first episode psychosis patients: One-year follow-up study. Journal of Affective Disorders, 256, 176-182. https://doi.org/10.1016/j.jad.2019.05. 078

Channon, S. (2004). Frontal lobe dysfunction and everyday problem-solving: Social and non-social contributions. Acta Psychologica, 115, 235-254. https://doi.org/10.1016/j.actpsy.2003.12. 008

Chu, C., Buchman-Schmitt, J.M., Stanley, I.H., Hom, M.A., Tucker, R. P., Hagan, C. R. et al. (2017). The interpersonal theory of suicide: A systematic review and meta-analysis of a decade of cross-national research. Psychological Bulletin, 143, 13131345. https://doi.org/10.1037/bul0000123

Cusi, A. M., Nazarov, A., Holshausen, K., Macqueen, G. M. \& McKinnon, M.C. (2012). Systematic review of the neural basis of social cognition in patients with mood disorders. Journal of Psychiatry \& Neuroscience, 37, 154-169. https://doi.org/10.1503/jpn.1 00179

Davis, M.H. (1980). A multidimensional approach to interindividual differences in empathy. JSAS Catalog of Selected Documents in Psychology, 10, 85.

Depp, C.A., Villa, J., Schembari, B.C., Harvey, P.D. \& Pinkham, A. (2018). Social cognition and short-term prediction of suicidal ideation in schizophrenia. Psychiatry Research, 270, 13-19. https://doi.org/10.1016/j.psychres.2018.09.005

Derntl, B., Finkelmeyer, A., Toygar, T. K., Hülsmann, A., Schneider, F., Falkenberg, D. i. et al. (2009). Generalized deficit in all core components of empathy in schizophrenia. Schizophrenia Research 108, 197-206. https://doi.org/10.1016/j.schres.2008.11.009

Derntl, B., Seidel, E. M., Schneider, F. \& Habel, U. (2012). How specific are emotional deficits? A comparison of empathic abilities in schizophrenia, bipolar and depressed patients. Schizophrenia Research, 142, 58-64. https://doi.org/10.1016/j.schres.2012.0 9.020

Dolcos, F., Katsumi, Y., Moore, M., Berggren, N., de Gelder, B., Derakshan, N. et al. (2020). Neural correlates of emotion-attention interactions: From perception, learning, and memory to social cognition, individual differences, and training interventions. Neuroscience and Biobehavioral Reviews, 108, 559-601. https://doi.org/10.1016/j.neubiorev.2019.08.017

Duñó, R., Pousa, E., Miguélez, M., Montalvo, I., Suarez, D. \& Tobeña, A. (2009). Suicidality connected with mentalizing anomalies in schizophrenia: A study with stabilized outpatients. Annals of the New York Academy of Sciences, 1167, 207-211. https://doi. org/10.1111/j.1749-6632.2009.04602.x

Dziobek, I., Fleck, S., Kalbe, E., Rogers, K., Hassenstab, J., Brand, M. et al. (2006). Introducing MASC: A movie for the assessment of social cognition. Journal of Autism and Developmental Disorders, 36, 623-636. https://doi.org/10.1007/s10803-006-010 7-0

D’Zurilla, T.J., Chang, E.C., Nottingham, E.J. \& Faccini, L. (1998). Social problem-solving deficits and hopelessness, depression, and suicidal risk in college students and psychiatric inpatients. Journal of Clinical Psychology, 54, 1091-1107. https://doi. org/10.1002/(SICI)1097-4679(199812)54:8<1091::AID-JCLP9> 3.0.CO;2-J

Erlangsen, A., Stenager, E., Conwell, Y., Andersen, P. K., Hawton, K., Benros, M.E. et al. (2020). Association between neurological disorders and death by suicide in Denmark. JAMA, 323, 444454. https://doi.org/10.1001/jama.2019.21834 
Eslinger, P.J., Moore, P., Anderson, C. \& Grossman, M. (2011). Social cognition, executive functioning, and neuroimaging correlates of empathic deficits in frontotemporal dementia. Journal of Neuropsychiatry and Clinical Neurosciences, 23, 74-82. https:// doi.org/10.1176/jnp.23.1.jnp74

Forkmann, T., Brähler, E., Gauggel, S. \& Glaesmer, H. (2012). Prevalence of suicidal ideation and related risk factors in the German general population. Journal of Nervous and Mental Disease, 200, 401-405. https://doi.org/10.1097/NMD.0b013e3182532 2cf

Gaesser, B. (2020). Episodic mindreading: Mentalizing guided by scene construction of imagined and remembered events. Cognition, 203. https://doi.org/10.1016/j.cognition.2020.104325

Gallant, C. \& Good, D. (2020). Examining the „reading the mind in the eyes test" as an assessment of subtle differences in affective theory of mind after concussion. Clinical Neuropsychologist, 34, 296-317. https://doi.org/10.1080/13854046.2019.1612946

Gallese, V. (2003). The roots of empathy: The shared manifold hypothesis and the neural basis of intersubjectivity. Psychopathology, 36, 171-180. https://doi.org/10.1159/000072786

Gournellis, R., Tournikioti, K., Touloumi, G., Thomadakis, C., Michalopoulou, P.G., Christodoulou, C. et al. (2018). Psychotic (delusional) depression and suicidal attempts: A systematic review and meta-analysis. Acta Psychiatrica Scandinavica, 137, 18-29. https://doi.org/10.1111/acps.12826

Gur, R.C., Ragland, J. D., Moberg, P.J., Turner, T.H., Bilker, W. B., Kohler, C. et al. (2001). Computerized neurocognitive scanning: I. Methodology and validation in healthy people. Neuropsychopharmacology, 25, 766-776. https://doi.org/10.1016/S0893133X(01)00278-0

Happé, F.G.E. (1994). An advanced test of theory of mind: Understanding of story characters' thoughts and feelings by able autistic, mentally handicapped and normal children and adults. Journal of Autism and Developmental Disorders, 24, 129-154.

Harenski, C.L., Brook, M., Kosson, D.S., Bustillo, J.R., Harenski, K.A., Caldwell, M.F. et al. (2017). Socio-neuro risk factors for suicidal behavior in criminal offenders with psychotic disorders. Social Cognitive and Affective Neuroscience, 12, 70-80. https://doi.org/10.1093/scan/nsw164

Hatkevich, C., Venta, A. \& Sharp, C. (2019). Theory of mind and suicide ideation and attempt in adolescent inpatients. Journal of Affective Disorders, 256, 17-25. https://doi.org/10.1016/j. jad.2019.05.051

Heilbron, N., Franklin, J.C., Guerry, J.D. \& Prinstein, M.J. (2014). Social and ecological approaches to understanding suicidal behaviors and nonsuicidal self-injury. Oxford Handbook of Suicide and Self-Injury, 206-234.

Isaacowitz, D. M., Livingstone, K. M. \& Castro, V.L. (2017). Aging and emotions: Experience, regulation, and perception. Current Opinion in Psychology, 17, 79-83. https://doi.org/10.1016/j. copsyc.2017.06.013

Joiner, T. (2005). Why people die by suicide. Harvard: University Press.

Kennedy, D.P. \& Adolphs, R. (2012). The social brain in psychiatric and neurological disorders. Trends in Cognitive Sciences, 16, 559-572. https://doi.org/10.1016/j.tics.2012.09.006

Kornreich, C., Philippot, P., Foisy, M. L., Blairy, S., Raynaud, E., Dan, B. et al. (2002). Impaired emotional facial expression recognition is associated with interpersonal problems in alcoholism. Alcohol and Alcoholism, 37, 394-400. https://doi.org/10.1093/ alcalc/37.4.394

Klonsky, E.D. \& May, A.M. (2015) The Three-Step Theory (3ST): A new theory rooted in the „ideation-to-action" framework. International Journal of Cognitive Therapy, 8, 114-129.

Kwok, S.Y. (2014). The moderating role of emotional competence in suicidal ideation among Chinese university students. Journal of
Advanced Nursing, 70, 843-854. https://doi.org/10.1111/ jan.12246

Lindenmayer, J.P., McGurk, S. R., Khan, A., Kaushik, S., Thanju, A., Hoffman, L. et al. (2013). Improving social cognition in schizophrenia: A pilot intervention combining computerized social cognition training with cognitive remediation. Schizophrenia Bulletin, 39, 507-517. https://doi.org/10.1093/schbul/sbs120

McClelland, H., Evans, J.J., Nowland, R., Ferguson, E. \& O'Connor, R.C. (2020). Loneliness as a predictor of suicidal ideation and behaviour: A systematic review and meta-analysis of prospective studies. Journal of Affective Disorders, 274, 880-896. https:// doi.org/10.1016/j.jad.2020.05.004

Monteleone, P., Cascino, G., Monteleone, A. M., Rocca, P., Rossi, A., Bertolino, A. et al. (2021). Prevalence of antipsychotic-induced extrapyramidal symptoms and their association with neurocognition and social cognition in outpatients with schizophrenia in the „real-life“. Progress in Neuro-Psychopharmacology \& Biological Psychiatry, 109. https://doi.org/10.1016/j.pnpbp.2021.110 250

Naghavi, M. \& Global Burden of Disease Self-Harm Collaborators. (2019). Global, regional, and national burden of suicide mortality 1990 to 2016: Systematic analysis for the Global Burden of Disease Study 2016. BMJ, 364. https://doi.org/10.1136/bmj.194

Nock, M., Nock, M.K., Borges, G. \& Ono, Y. (Eds.). (2012). Suicide: Global perspectives from the WHO world mental health surveys. Cambridge: University Press.

O'Connor, R.C. \& Kirtley, O.J. (2018). The integrated motivationalvolitional model of suicidal behaviour. Philosophical Transactions of the Royal Society of London. Series B, Biological Sciences, 373. https://doi.org/10.1098/rstb.2017.0268

Ortuño-Sierra, J., Aritio-Solana, R., Del Casal, A. \& Fonseca-Pedrero, E. (2020). Neurocognitive functioning in adolescents at risk for suicidal behaviors. Archives of Suicide Research, 1-15. https://doi.org/10.1080/13811118.2020.1746938

Paradiso, S., Beadle, J. N., Raymont, V. \& Grafman, J. (2016). Suicidal thoughts and emotion competence. Journal of Clinical and Experimental Neuropsychology, 38, 887-899. https://doi.org/10. 1080/13803395.2016.1172558

Penn, D.L., Roberts, D.L., Combs, D. \& Sterne, A. (2007). Best practices: The development of the Social Cognition and Interaction Training program for schizophrenia spectrum disorders. Psychiatric Services, 58, 449-451. https://doi.org/10.1176/ps. 2007.58.4.449

Posner, K., Brodsky, B., Yershova, K., Buchanan, J. \& Mann, J. (2014). The classification of suicidal behavior. In M. Nock (Ed.), The Oxford Handbook of Suicide and Self-Injury (pp. 7-22). Oxford: University Press.

Preston, S.D. \& de Waal, F.B. (2002). Empathy: Its ultimate and proximate bases. Behavioral and Brain Sciences, 25, 1-71. https://doi.org/10.1017/s0140525×02000018

Richard-Devantoy, S., Guillaume, S., Olié, E., Courtet, P. \& Jollant, F. (2013). Altered explicit recognition of facial disgust associated with predisposition to suicidal behavior but not depression. Journal of Affective Disorders, 150, 590-593. https://doi. org/10.1016/j.jad.2013.01.049

Seymour, K. E., Jones, R. N., Cushman, G. K., Galvan, T., Puzia, M.E., Kim, K. L. et al. (2016). Emotional face recognition in adolescent suicide attempters and adolescents engaging in non-suicidal self-injury. European Child \& Adolescent Psychiatry, 25, 247259. https://doi.org/10.1007/s00787-015-0733-1

Shamay-Tsoory, S. G. (2011). The neural bases for empathy. Neuroscientist, 17, 18-24. https://doi.org/10.1177/1073858410379268

Shamay-Tsoory, S.G. \& Aharon-Peretz, J. (2007). Dissociable prefrontal networks for cognitive and affective theory of mind: a lesion study. Neuropsychologia, 45, 3054-3067. https://doi. org/10.1016/j.neuropsychologia.2007.05.021 
Shamay-Tsoory, S. G., Aharon-Peretz, J. \& Perry, D. (2009). Two systems for empathy: A double dissociation between emotional and cognitive empathy in inferior frontal gyrus versus ventromedial prefrontal lesions. Brain, 132, 617-627. https://doi. org/10.1093/brain/awn279

Speckens, A. E. \& Hawton, K. (2005). Social problem solving in adolescents with suicidal behavior: A systematic review. Suicide \& Life-Threatening Behavior, 35, 365-387. https://doi.org/10.1521/ suli.2005.35.4.365

Statistisches Bundesamt. (2021). Todesursachen. Verfügbar unter http://www.destatis.de

Szanto, K., Dombrovski, A.Y., Sahakian, B.J., Mulsant, B. H., Houck, P. R., Reynolds, C. F. et al. (2012). Social emotion recognition, social functioning, and attempted suicide in late-life depression. American Journal of Geriatric Psychiatry, 20, 257-265. https:// doi.org/10.1097/JGP.0b013e31820eea0c

Thoma, P., Friedmann, C. \& Suchan, B. (2013). Empathy and social problem solving in alcohol dependence, mood disorders and selected personality disorders. Neuroscience and Biobehavioral Reviews, 37, 448-470. https://doi.org/10.1016/j.neubiorev.201 3.01 .024

Tsai, A.C., Lucas, M. \& Kawachi, I. (2015). Association between social integration and suicide among women in the United States. JAMA Psychiatry, 72, 987-993. https://doi.org/10.1001/jamapsychiatry.2015.1002

Tsai, A.C., Lucas, M., Sania, A., Kim, D. \& Kawachi, I. (2014). Social integration and suicide mortality among men: 24-year cohort study of U.S. health professionals. Annals of Internal Medicine, 161, 85-95. https://doi.org/10.7326/M13-1291

Tsypes, A., Burkhouse, K.L. \& Gibb, B.E. (2016). Classification of facial expressions of emotion and risk for suicidal ideation in children of depressed mothers: Evidence from crosssectional and prospective analyses. Journal of Affective Disorders, 197, 147-150. https://doi.org/10.1016/j.jad.2016.0 3.037

Vaquero-Lorenzo, C. \& Vasquez, M.A. (2020). Suicide: Genetics and heritability. Current Topics in Behavioral Neurosciences, 46, 6378. https://doi.org/10.1007/7854_2020_161

Villa, J., Pinkham, A.E., Kaufmann, C.N., Granholm, E., Harvey, P.D. \& Depp, C.A. (2018). Interpersonal beliefs related to suicide and facial emotion processing in psychotic disorders. Journal of Psychiatric Research, 100, 107-112. https://doi.org/10.1016/j. jpsychires.2018.02.016
Vucurovic, K., Caillies, S. \& Kaladjian, A. (2020). Neural correlates of theory of mind and empathy in schizophrenia: An activation likelihood estimation meta-analysis. Journal of Psychiatric Research, 120,163-174. https://doi.org/10.1016/j.jpsychires.2019.10.018

Vytal, K. \& Hamann, S. (2010). Neuroimaging support for discrete neural correlates of basic emotions: A voxel-based meta-analysis. Journal of Cognitive Neuroscience, 22, 2864-2885. https:// doi.org/10.1162/jocn.2009.21366

Waern, M., Rubenowitz, E. \& Wilhelmson, K. (2003). Predictors of suicide in the old elderly. Gerontology, 49, 328-334. https://doi. org/10.1159/000071715

Wang, W., Zhou, Y., Wang, J., Xu, H., Wei, S., Wang, D. et al. (2020). Prevalence, clinical correlates of suicide attempt and its relationship with empathy in patients with schizophrenia. Progress in Neuro-Psychopharmacology \& Biological Psychiatry, 99. https://doi.org/10.1016/j.pnpbp.2020.109863

Ze, O., Thoma, P. \& Suchan, B. (2014). Cognitive and affective empathy in younger and older individuals. Aging \& Mental Health, 18,929-935. https://doi.org/10.1080/13607863.2014.899973

\section{Historie}

Eingereicht: 12. September 2020

Akzeptiert: 12. März 2021

\section{Förderung}

Open-Access-Veröffentlichung ermöglicht durch

Ruhr-Universität Bochum.

\section{Interessenkonflikt}

Alle Autor_innen geben keinen Interessenkonflikt an.

\section{PD Dr. Patrizia Thoma}

Neuropsychologisches Therapie Centrum

AG Klinische Neuropsychologie

Fakultät für Psychologie

Ruhr-Universität Bochum

Universitätsstr. 150

44799 Bochum

Deutschland

patrizia.thoma@rub.de 